\title{
The Added Mass Coefficient computation of sphere, ellipsoid and marine propellers using Boundary Element Method
}

\author{
Hassan Ghassemi, Assoc. Prof. \\ Amirkabir University of Technology \\ Ehsan Yari, M. Sc. \\ Malekashtar University of Technology
}

\begin{abstract}
Added mass is an important and effective dynamic coefficient in accelerating, non uniform motion as a result of fluid accelerating around a body. It plays an important role, especially in vessel roll motion, control parameters as well as in analyzing the local and global vibration of a vessel and its parts like propellers and rudders. In this article, calculating the Added Mass Coefficient has been examined for a sphere, ellipsoid, marine propeller and hydrofoil; using numerical Boundary Element Method. Since an Ellipsoid and a sphere have simple geometric shapes and the Analytical values of their added mass coefficients are available, so that the results of added mass matrix are obtained and evaluated, using the boundary element method. Then the added mass matrix is computed in a given geometrical and flow specifications for a specific propeller and its results are studied versus experimental results, which it's current numerical data In comparison with other numerical methods has a good conformity with experimental results. The most important advantage of the method in determining the added mass matrix coefficients for the surface and underwater vessels and the marine propellers is extracting all the added mass coefficients with very good Accuracy, while in other numerical methods it is impossible to extract all the coefficients with the Desired Accuracy.
\end{abstract}

Key words: Added mass coefficient; Boundary element method; Marine propeller; Ellipsoid

\section{INTRODUCTION}

In fluid mechanics, added mass or virtual mass, is the added inertia to the system, owing to that the Increase or decrease in the body acceleration should cause the Fluid to move around the body in such a way that the object can move through it, because the body and the fluid cannot simultaneously occupy this physical space. For simplicity this can be modeled in such a way that the Volume of fluid moves along the object, While in fact all parts of the fluid move with varying degrees. Added mass coefficient matrix usually is expressed as a dimensionless value which is equal to added mass coefficient divided by the Mass of displaced fluid. For example the body density can be considered in the body volume. In general, the added mass coefficient is a second order tensor that depends on the fluid acceleration vector in order to obtain force vector on the body.

The added mass was considered as the first time in 1776 by Dubua who did laboratory studies on spherical pendulum in low swings. [2] Precise mathematical equations for the added mass of sphere, was obtained by Green (1833) and Stokes (1843) respectively. Stokes, also studied on the sphere motions in a finite volume of fluid. Later, following the efforts of many researchers, the concept of added mass was generalized for moving a desired object in different flow regimes. [3]

\section{THE ADDED MASS COEFFICIENT RELATIONS}

The hydrodynamic forces and moments are determined by fluid inertia and viscous properties according to the motion of a body in real incompressible fluid. Definitely, the other forces and moments can be calculated by obtaining one of the inertia or viscous terms. Since the fluid can be assumed as an ideal one (Inviscid). Therefore the inertia forces and moments can be calculated with this estimate. The forces and moments of inertia can be introduced as the body added mass terms, using this method.

Assume that the body with surface $\mathrm{S}$ is moving in an infinite homogeneous ideal Fluid. Consider two systems of coordinates: the stationary one (We denote it by XYZ) and the coordinate system moving together with the body (We denote it by Oxyz). The vortex-free condition implies the existence of a potential $\phi(X, Y, Z, t)$, assuming that the moment of these two systems of coordinate are specified. So the components of the fluid velocity are defined as follows:

$$
\mathrm{v}_{\mathrm{X}}=\frac{\partial \varphi}{\partial \mathrm{X}} ; \mathrm{v}_{\mathrm{Y}}=\frac{\partial \varphi}{\partial \mathrm{Y}} ; \mathrm{v}_{\mathrm{Z}}=\frac{\partial \varphi}{\partial \mathrm{Z}}
$$


Using the continuity and incompressibility of fluid and due to the vortex-free condition as mentioned above the Laplace equation is implied as follows:

$$
\frac{\partial^{2} \varphi}{\partial \mathrm{X}^{2}}+\frac{\partial^{2} \varphi}{\partial \mathrm{Y}^{2}}+\frac{\partial^{2} \varphi}{\partial \mathrm{Z}^{2}}=0 ; \nabla^{2} \phi=0
$$

The boundary conditions for Eq. (2) look as follows:

1. The watertight condition, valid on the surface $S$ :

$$
\left.\frac{\partial \varphi}{\partial \mathrm{n}}\right|_{\mathrm{S}}=\mathrm{u}_{\mathrm{n}}
$$

Where $\left(\left.\frac{\partial \varphi}{\partial n}\right|_{S}=u_{n}\right)$ is a projection of fluid velocity on the (external) direction $n$, which is normal to surface $S$; $u$ is the velocity projection of a point of the body, normal to surface $n$.

2. Stationary condition at infinity:

$$
\underset{r \rightarrow \infty}{\operatorname{Lim}} \frac{\partial \varphi}{\partial X}=\operatorname{Lim}_{r \rightarrow \infty} \frac{\partial \varphi}{\partial Y}=\operatorname{Lim}_{r \rightarrow \infty} \frac{\partial \varphi}{\partial Z}=0
$$

Where $\mathrm{r}^{2}=\mathrm{X}^{2}+\mathrm{Y}^{2}+\mathrm{Z}^{2}$ ( $\mathrm{r}$ is the distance from the origin to a fluid point). The function $\phi$ vanishes at infinity as $1 / \mathrm{r}^{2}$, whereas its first-order coordinate derivatives vanish as $1 / \mathrm{r}^{3}[4,5]$

From the formulation of the problem (1-4) it is seen that the function $\phi$ depends on time $t$ via the boundary condition of right-hand side equation (3), so the boundary condition is investigated specifically. The origin $\mathrm{O}$ is chosen to coincide with an arbitrary point of the body

The velocity of the point $\mathrm{O}$ is Denoted by $\overrightarrow{\mathrm{u}}_{0}$ (components $\mathrm{u}_{0 \mathrm{x}}, \mathrm{u}_{0 \mathrm{y}}, \mathrm{u}_{0 \mathrm{z}}$ are projections of the vector $\mathrm{u}_{0}$ on coordinate axes attached to the body).

The angular velocity of the body with respect to the point $\mathrm{O}$ is also donated by $\vec{\omega}$ (components of $\vec{\omega}$ in the same coordinate system are denoted by $\left.\omega_{v}, \omega_{y}, \omega_{z}\right)$. Thus, the velocity of an arbitrary point of the body, including any point of its surface $\mathrm{S}$, is determined by the following equation:

$$
\overrightarrow{\mathrm{u}}=\overrightarrow{\mathrm{u}}_{0}+\overrightarrow{\mathrm{\omega}} \times \overrightarrow{\mathrm{r}}
$$

Where $\vec{r}$ is the vector, determining the position of the point. The components of Eq. (5) look as follows:

$$
\begin{aligned}
& u_{x}=u_{0 x}+\omega_{y} z-\omega_{z} y \\
& u_{y}=u_{0 y}+\omega_{z} x-\omega_{x} z \\
& u_{z}=u_{0 z}+\omega_{x} y-\omega_{y} x
\end{aligned}
$$

On the surface $\mathrm{S}$ we have:

$$
u_{n}=u_{x} \cos (n, x)+u_{y} \cos (n, y)+u_{z} \cos (n, z)
$$

Writing: $\alpha \equiv \cos (\mathrm{n}, \mathrm{x}) ; \beta \equiv \cos (\mathrm{n}, \mathrm{y}) ; \gamma \equiv \cos (\mathrm{n}, \mathrm{z})$ and substituting (6) into (7), the following form of the boundary condition is obtained (3):

$$
\begin{aligned}
\left.\frac{\partial \varphi}{\partial \mathrm{n}}\right|_{\mathrm{S}}=\mathrm{u}_{\mathrm{n}} & =\mathrm{u}_{0 \mathrm{x}} \alpha+\mathrm{u}_{0 \mathrm{y}} \beta+\mathrm{u}_{0 \mathrm{z}} \gamma+\omega_{\mathrm{x}}(\mathrm{y} \gamma-\mathrm{z} \beta)+ \\
& +\omega_{\mathrm{y}}(\mathrm{z} \alpha-\mathrm{x} \gamma)+\omega_{\mathrm{z}}(\mathrm{x} \beta-\mathrm{y} \alpha)
\end{aligned}
$$

In the formula (8) the variables $\alpha, \beta, \gamma, y \gamma-z \beta, z \alpha-x \gamma$, $\mathrm{x} \beta-\mathrm{y} \alpha$ are determined only based on the shape of the body surface. The body motion and the dynamics of the flow are determined by the functions $u_{0 x}, u_{0 y}, u_{0 z}, \omega_{x}, \omega_{y}, \omega_{z}$. The potential $\phi$ is represented as follows, due to the Linearity of the problem.

$$
\varphi=u_{0 x} \varphi_{1}+u_{0 y} \varphi_{2}+u_{0 z} \varphi_{3}+\omega_{x} \varphi_{4}+\omega_{y} \varphi_{5}+\omega_{z} \varphi_{6}
$$

In the above equation $f_{i}, i=1,2,3$ are the flow potentials corresponding to the body moving along the axes $\mathrm{x}, \mathrm{y}, \mathrm{z}$ at unit linear velocities, respectively. On the other hand $\phi_{i}, i=4,5,6$ are potentials, corresponding to rotation of the body around the same axes at unit angular velocities respectively. It is seen that the body motion in an ideal infinite fluid causes to solve six problems. The first problem can be formulated as follows: the solution of the Laplace equation $\Delta \phi_{1}=0$ will be found, using the following boundary conditions:

$$
\begin{gathered}
\frac{\partial \phi_{1}}{\partial \mathrm{n}}=\alpha \\
\frac{\partial \phi_{1}}{\partial \mathrm{X}}+\frac{\partial \phi_{1}}{\partial \mathrm{Y}}+\frac{\partial \phi_{1}}{\partial \mathrm{Z}} \rightarrow 0 \text { as } \mathrm{r} \rightarrow \infty
\end{gathered}
$$

The function $\frac{\partial \phi_{1}}{\partial \mathrm{n}}=\alpha$ is given on the surface $\mathrm{S}$ and The last (sixth) problem, taking (8) into account. To solve the problem, considering the Laplace equation $\Delta \phi_{6}=0$ using the following boundary conditions: $\frac{\partial \phi_{6}}{\partial \mathrm{n}}=\mathrm{x} \beta-\mathrm{y} \alpha$ which is given at the surface $\mathrm{S}$, so:

$$
\frac{\partial \phi_{6}}{\partial \mathrm{X}}+\frac{\partial \phi_{6}}{\partial \mathrm{Y}}+\frac{\partial \phi_{6}}{\partial \mathrm{Z}} \rightarrow 0 \text { as } \mathrm{r} \rightarrow \infty
$$

It can be seen that the function $\phi_{i}, i=1,2, \ldots 6$ does not depend on $\mathrm{u}_{0}$ and $\omega_{0}$. These functions are determined only by the shape of the surface $\mathrm{S}$ of the body and Choice of coordinate system Oxyz attached to the body.

\section{KINETIC ENERGY OF THE FLUID}

In order to to obtain fluid kinetic energy, considering the surface $\mathrm{S}$ and the sphere $\Sigma$ of radius a including the body with surrounding Fluid, the following integral is defined.

$$
\begin{gathered}
T=\frac{1}{2} \rho \iiint_{V} v^{2} d V= \\
=\frac{1}{2} \rho \iiint_{V}\left[\left(\frac{\partial \phi}{\partial x}\right)^{2}+\left(\frac{\partial \phi}{\partial y}\right)^{2}+\left(\frac{\partial \phi}{\partial z}\right)^{2}\right] d x d y d z
\end{gathered}
$$

Where $\rho$, is the fluid Density

Using Green's transformation for two functions $\left(\phi_{1}, \phi_{2}\right)$ :

$$
\iiint_{V}\left(\frac{\partial \phi_{1}}{\partial x} \frac{\partial \phi_{2}}{\partial x}+\frac{\partial \phi_{1}}{\partial y} \frac{\partial \phi_{2}}{\partial y}+\frac{\partial \phi_{1}}{\partial z} \frac{\partial \phi_{2}}{\partial z}\right) d x d y d z=
$$$$
=-\iint_{\mathrm{S}+\Sigma} \phi_{1} \frac{\partial \phi_{2}}{\partial \mathrm{n}} \mathrm{dS}-\iiint_{\mathrm{V}} \phi_{1}\left(\frac{\partial^{2} \phi_{2}}{\partial \mathrm{x}^{2}}+\frac{\partial^{2} \phi_{2}}{\partial \mathrm{y}^{2}}+\frac{\partial^{2} \phi_{2}}{\partial \mathrm{z}^{2}}\right) \mathrm{dxdydz}
$$

And considering $\phi_{1}=\phi_{2}=\phi, \Delta \phi=0$ and putting words in the above equation the following expression is gotten:

$$
\mathrm{T}=-\frac{\rho}{2} \iint_{\mathrm{S}} \phi \frac{\partial \phi}{\partial \mathrm{n}} \mathrm{dS}-\frac{\rho}{2} \iint_{\Sigma} \phi \frac{\partial \phi}{\partial \mathrm{n}} \mathrm{dS}
$$

The Second term in the right hand side of Eq. (14) tends to zero as $\alpha \rightarrow \infty$ according to the asymptotic behavior of $\phi$ and its first derivatives while $r \rightarrow \infty$. Therefore, the following formula is obtained for the total kinetic energy of the fluid around the surface $\mathrm{S}$ :

$$
\mathrm{T}=-\frac{\rho}{2} \iint_{\mathrm{S}} \phi \frac{\partial \phi}{\partial \mathrm{n}} \mathrm{dS}
$$


Substituting the expression (9) in to (15) and writing:

$$
\begin{gathered}
\mathrm{u}_{0 \mathrm{x}}=\mathrm{u}_{1}, \mathrm{u}_{0 \mathrm{y}}=\mathrm{u}_{2}, \mathrm{u}_{0 \mathrm{y}}=\mathrm{u}_{3}, \\
\omega_{\mathrm{x}}=\mathrm{u}_{4}, \omega_{\mathrm{y}}=\mathrm{u}_{5}, \omega_{\mathrm{z}}=\mathrm{u}_{6}
\end{gathered}
$$

The expression (16) is finally obtained:

$$
\mathrm{T}=\frac{1}{2} \sum_{\mathrm{i}=1}^{6} \sum_{\mathrm{k}=1}^{6} \lambda_{\mathrm{ik}} \mathrm{u}_{\mathrm{i}} \mathrm{u}_{\mathrm{k}}
$$

Where $\lambda_{\text {ik }}$ is:

$$
\lambda_{\mathrm{ik}}=-\rho \iint_{\mathrm{S}} \frac{\partial \phi_{\mathrm{i}}}{\partial \mathrm{n}} \phi_{\mathrm{k}} \mathrm{dS}
$$

$\lambda_{\mathrm{ik}}$ is called the added mass of the body.

According to Eq. (17) it is found that the added mass, do not depend on the body velocity and Kinematics of the motion but is only a function of the body surface geometry.

Applying Green's formula to functions $\phi_{i}$ and $\phi_{k}$ in the volume $\mathrm{V}$ between the surfaces $\Sigma$ and $\mathrm{S}$ then:

$$
\iiint_{\mathrm{V}}\left(\phi_{\mathrm{i}} \Delta \phi_{\mathrm{k}}-\phi_{\mathrm{k}} \Delta \phi_{\mathrm{i}}\right) \mathrm{dV}=
$$

$=\iint_{\Sigma}\left(\phi_{\mathrm{i}} \frac{\partial \phi_{\mathrm{k}}}{\partial \mathrm{n}}-\phi_{\mathrm{k}} \frac{\partial \phi_{\mathrm{i}}}{\partial \mathrm{n}}\right) \mathrm{d} \mathrm{S}-\iint_{\mathrm{S}}\left(\phi_{\mathrm{i}} \frac{\partial \phi_{\mathrm{k}}}{\partial \mathrm{n}}-\phi_{\mathrm{k}} \frac{\partial \phi_{\mathrm{i}}}{\partial \mathrm{n}}\right) \mathrm{dS}$

Since $\Delta \phi_{\mathrm{i}}=\Delta \phi_{\mathrm{k}}=0$, so that the left-hand side of the equation equals zero, and the first term of the right-hand side of the equation tends to zero at $\alpha \rightarrow \infty$. Therefore, the condition for the infinite fluid surrounding the body is as follows:

$$
\iint_{\mathrm{S}} \phi_{\mathrm{i}} \frac{\partial \phi_{\mathrm{k}}}{\partial \mathrm{n}} \mathrm{dS}=\iint_{\mathrm{S}} \phi_{\mathrm{k}} \frac{\partial \phi_{\mathrm{i}}}{\partial \mathrm{n}} \mathrm{dS}
$$

Since $\lambda_{\mathrm{ik}}=\lambda_{\mathrm{ki}}$. Therefore, only 21 values out of $36 \lambda_{\mathrm{ik}}(\mathrm{i}, \mathrm{k}=$ $=1,2 \ldots 6)$ are independent.

This part, in fact proved that the added mass matrix is symmetric.

To obtain the added mass coefficient matrix using force view, ultimately the same formula is achieved. [6]

\section{CALCULATING THE ADDED MASS COEFFICIENT USING THE BOUNDARY ELEMENT METHOD}

Boundary element method is based on the Green's theory. Based on the theory solving the fluid flow around the body can be transferred from the volume solving domain around the body to the body boundary that is performed on the produced surface elements in three-dimensional modes. The boundary element method is used to obtain all six unit potentials corresponding to the six free degrees. The added mass tensor can be computed, obtaining the unit potential. The forces and momentum also can be achieved, having the added mass. [7]

$$
\int_{\Omega} \nabla \cdot \mathrm{ud} \Omega=\int_{\Gamma} \mathrm{u} \cdot \mathrm{nd} \mathrm{ds}
$$

\section{THE BOUNDARY ELEMENT METHOD TO COMPUTE THE ADDED MASS COEFFICIENT}

Consider the Closed area $\Omega$, the boundary $\mathrm{S}$ and unit vector $\vec{n}$ normal to S (Fig. 1). The boundary $\mathrm{S}$ is composed of the body wetted surface SB, the wake surface SW and the external control surface $\mathrm{S}_{\infty}$ including surface area $\mathrm{SB}, \mathrm{SW}$.

The $\Omega$ encountered the Uniform inflow velocity $\vec{V}_{\mathrm{l}}$, assuming the flow is Incompressible, Inviscid and Irrotational in the domain $\Omega$, in the upstream flow.

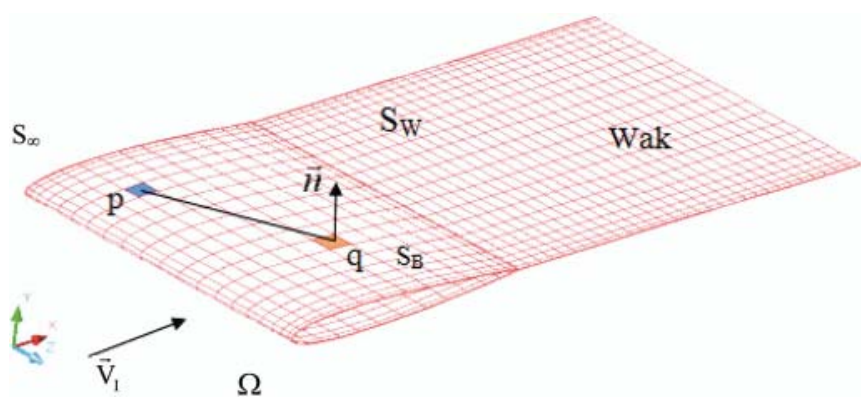

Fig. 1. Application of Green theory for hydrofoils

With these assumptions, the flow field around the body can be identified using Perturbation Velocity potential $\phi$, which satisfies the Laplace equation:

$$
\nabla^{2} \phi=0
$$

To solving the problem, the boundary conditions are considered as follows:

\section{Kinematic Boundary Condition}

Based on the Kinematic boundary condition, the Flow velocity normal to the body surface must be zero.

$$
\frac{\partial \Phi}{\partial \mathrm{n}}=0 \rightarrow \frac{\partial \phi}{\partial \mathrm{n}}=-\overrightarrow{\mathrm{V}}_{\mathrm{I}} \cdot \overrightarrow{\mathrm{n}}
$$

Where $\vec{n}$, is the unit vector normal to outside of the boundary [8] and is changed as follows, considering the unit potential Kinematic of Boundary Condition:

$$
\left.\frac{\partial \phi_{j}}{\partial \mathrm{n}}\right|_{\mathrm{j}=1 \rightarrow 6}=\left\{\begin{array}{l}
\left.\mathrm{n}_{\mathrm{i}}\right|_{\mathrm{i}=1 \rightarrow 3} ^{\mathrm{j}=1 \rightarrow 3} \\
\left(\mathrm{r} \times \mathrm{n}_{\mathrm{i}}\right)_{\mathrm{i}=1 \rightarrow 3}^{\mathrm{j}=4 \rightarrow 6}
\end{array}\right.
$$
[12].

Where, $\mathrm{r}$ is a vertical distance from a fixed origin point

\section{Kinematic And Dynamic Conditions In Wake}

Based on this condition, there is no velocity jump of flow on the wake vortex, however there is velocity jump of potential on the surface which is equal to circulation $\Gamma$ around the fin. The mathematical relations of flow velocity and potential are expressed as follows:

$$
\begin{gathered}
(\Delta \phi)_{\text {on }_{\mathrm{w}}}=\phi^{\mathrm{B}}-\phi^{\mathrm{F}}=\Gamma \\
\left(\Delta \frac{\partial \phi}{\partial \mathrm{n}}\right)=\left(\frac{\partial \phi}{\partial \mathrm{n}}\right)^{\mathrm{B}}-\left(\frac{\partial \phi}{\partial \mathrm{n}}\right)^{\mathrm{F}}=0
\end{gathered}
$$
[8].

Where B and F showing back and face sides of the propeller

\section{KUTTA CONDITION IN THE TRAILING EDGE}

Hess and Smith have shown that the flow passing along a thin and non lifting body can be described by the distribution singularities of source. But to describe the flow passing along lifting bodies, modeled by the circulation distribution on the surface, a boundary condition for the Trailing Edge of the body must be satisfied, expressing that the velocity at the trailing edge should be limited and unique. [9, 10]

$$
|\nabla \phi|_{\mathrm{TE}}<0
$$




\section{Surface At Infinity}

At the boundary surface at infinity $\mathrm{S}_{\infty}$, the perturbation velocity due to the body surface must tend to zero.

$$
\nabla \phi \rightarrow 0 \text { as } \mathrm{S}_{\infty} \rightarrow 0
$$

Using Green's theory, general answers of the Laplace equation for each point $\mathrm{P}$ can be expressed as the following integral equation:

$$
\begin{gathered}
4 \pi \phi(\mathrm{P})=\iint_{\mathrm{S}_{\mathrm{B}}} \phi(\mathrm{q}) \frac{\partial}{\partial \mathrm{n}_{\mathrm{q}}}\left(\frac{1}{\mathrm{R}(\mathrm{p}, \mathrm{q})}\right) \mathrm{ds}+ \\
-\iint_{\mathrm{S}_{\mathrm{B}}} \frac{\partial \phi}{\partial \mathrm{n}_{\mathrm{q}}}\left(\frac{1}{\mathrm{R}(\mathrm{p}, \mathrm{q})}\right) \mathrm{ds}+\iint_{\mathrm{S}_{\mathrm{w}}} \Delta \phi(\mathrm{q}) \frac{\partial}{\partial \mathrm{n}_{\mathrm{q}}}\left(\frac{1}{\mathrm{R}(\mathrm{p}, \mathrm{q})}\right) \mathrm{ds}
\end{gathered}
$$

In the above equation $\partial \phi / \partial \mathrm{n}$ is determined, using the boundary condition of $\mathrm{Eq}(23) .1 / \mathrm{R}(\mathrm{p}, \mathrm{q})$, is the distance between $\mathrm{p}$ and $\mathrm{q}$, so that the only remaining unknown for solving Eq (27) is $\phi$. [8]

\section{EQUATIONS DISCRETIZATION}

In order to solve $\mathrm{Eq}$ (27), the equation form must be discrete and the body boundary surface shall be made of tetragonal elements.

Fractional equations lead to linear algebraic equation system for $\phi$ and $i$ as follows:

$$
\begin{gathered}
2 \pi \phi_{\mathrm{i}}=\sum_{\mathrm{j}=1}^{\mathrm{N}} \mathrm{D}_{\mathrm{ij}}\left(\phi_{\mathrm{i}}\right)+\sum_{\mathrm{j}=1}^{\mathrm{N}_{\mathrm{R}}} \sum_{\mathrm{l}=1}^{\mathrm{N}_{\mathrm{W}}} \mathrm{W}_{\mathrm{ijl}}(\Delta \phi)_{\mathrm{i}}+ \\
+\sum_{\mathrm{j}=1}^{\mathrm{N}} \mathrm{S}_{\mathrm{ij}}\left(\frac{\partial \phi}{\partial \mathrm{n}}\right)_{\mathrm{j}} \quad \mathrm{i}=1,2, \ldots, \mathrm{N}_{\text {tot }}
\end{gathered}
$$

Where $\mathrm{D}_{\mathrm{ij}}, \mathrm{S}_{\mathrm{ij}}$, and $\mathrm{W}_{\mathrm{ijl}}$ are Dipole coefficients and source for $j$ element that acts on the arithmetic point $i$. The coefficients are defined as follows:

$$
\begin{aligned}
& \mathrm{D}_{\mathrm{ij}}=\sum_{\mathrm{k}=1}^{\mathrm{k}}\left[\iint_{\mathrm{S}_{\mathrm{B}}} \frac{\partial}{\partial \mathrm{n}_{\mathrm{j}}}\left(\frac{1}{\mathrm{R}_{\mathrm{ij}}}\right) \mathrm{ds}_{\mathrm{j}}\right]_{\mathrm{k}} \text { on } \mathrm{S}_{\mathrm{B}} \\
& \mathrm{W}_{\mathrm{ij} 1}=\sum_{\mathrm{k}=1}^{\mathrm{k}}\left[\iint_{\mathrm{S}_{\mathrm{W}}} \frac{\partial}{\partial \mathrm{n}_{\mathrm{j}}}\left(\frac{1}{\mathrm{R}_{\mathrm{ij}}}\right) \mathrm{ds}_{\mathrm{j}}\right]_{\mathrm{k}} \text { on } \mathrm{S}_{\mathrm{W}} \\
& \mathrm{S}_{\mathrm{ij}}=\sum_{\mathrm{k}=1}^{\mathrm{k}}\left[\iint_{\mathrm{S}_{\mathrm{B}}}\left(\frac{1}{\mathrm{R}_{\mathrm{ij}}}\right) \mathrm{d} \mathrm{s}_{\mathrm{j}}\right]_{\mathrm{k}} \text { on } \mathrm{S}_{\mathrm{B}}
\end{aligned}
$$

To obtain these coefficients the numerical solution of the integrals is used. Calculating these coefficients, the following matrix equation is formed

Solving the equations using the Gauss - Sydl method is done as a repetitive solving, so that $\phi_{\mathrm{j}}$ are obtained. [11]

$$
[\mathrm{D}][\phi]=[\mathrm{S}]+[\mathrm{W}][\Delta \phi]
$$

Where:

[D] = dipole Potential effects of induction coefficient matrix $[\mathrm{S}]=$ source Potential effects of induction coefficient matrix $[\mathrm{W}]=$ dipole Potential effects of induction coefficient matrix

For lifting and non-lifting bodies, the above computational method can be used, but the difference is in the use of Kutta boundary condition which there is no need to consider it in non-lifting bodies, so that the results are obtained directly regarding the kinematic boundary condition.
Although the Kutta condition is considered to analyze the propeller and the hydrofoil, the obtaining results have no significant difference with the ones which are obtained without considering the Kutta condition. So, regarding this issue it can be expressed that only the $\mathrm{D}$ and $\mathrm{S}$ out of the $\mathrm{D}, \mathrm{S}$ and $\mathrm{W}$ are important for calculating the added mass matrix.

$$
\begin{array}{rr}
D_{i j}=\sum_{k=1}^{k}\left[\iint_{S_{B}} \frac{\partial}{\partial n_{j}}\left(\frac{1}{R_{i j}}\right) d s_{j}\right]_{k} \quad \text { on } S_{B} \\
S_{i j}=\sum_{k=1}^{k}\left[\iint_{S_{B}}\left(\frac{1}{R_{i j}}\right) d s_{j}\right]_{k} & \text { on } S_{B}
\end{array}
$$

Which in Reference 12, the integrals are expressed as follows that in fact are the same general term in Eq (27) and with extracting the D and S, the formulas in Eq (31) is obtained.

$$
\begin{gathered}
\int\left[\frac{1}{\mathrm{R}} \frac{\partial \phi}{\partial \mathrm{n}}\right] \mathrm{d} \mathrm{S}=\left.\sum_{\substack{\mathrm{K}=1 \\
\mathrm{~K} \neq \mathrm{i}}}^{\mathrm{N}} \frac{1}{\mathrm{R}} \frac{\partial \phi_{\mathrm{j}}}{\partial \mathrm{n}}\right|_{\mathrm{j}=1 \rightarrow 6} \delta \mathrm{S}_{\mathrm{K}} \\
\int_{\mathrm{S}_{\mathrm{B}}}\left[\phi \frac{\partial}{\partial \mathrm{n}}\left(\frac{1}{\mathrm{R}}\right)\right] \mathrm{dS}=\sum_{\substack{\mathrm{K}=1 \\
\mathrm{~K} \neq \mathrm{i}}}^{\mathrm{N}}\left[\frac{\partial}{\partial \mathrm{n}}\left(\frac{1}{\mathrm{R}}\right)\right] \delta \mathrm{S}_{\mathrm{K}}+2 \pi \phi_{\mathrm{i}=\mathrm{K}}
\end{gathered}
$$

The Flowchart Of The Added Mass Matrix Extraction Using The Boundary Element Method

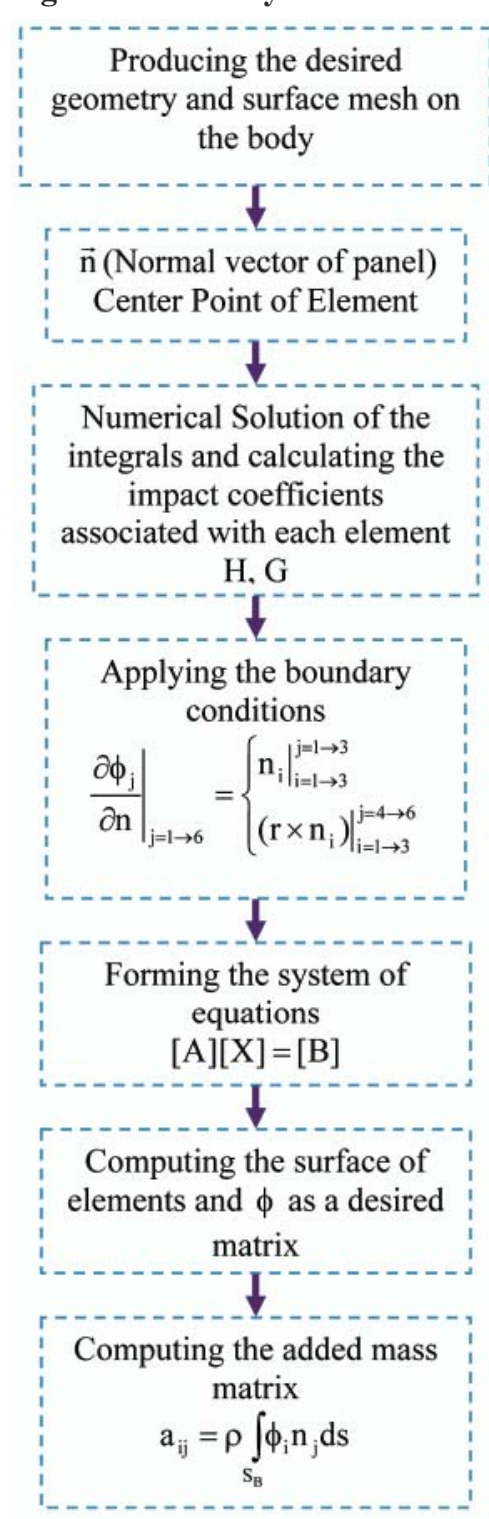


If the above integrals are formulated based on matrix equations, the following relation is obtained:

$$
\left[\mathrm{A}_{\mathrm{iK}}\right]\left[\phi_{\mathrm{K}}\right]=\left[\mathrm{B}_{\mathrm{i}}\right]
$$

So that $\mathrm{i}=\mathrm{K}$ and $\mathrm{A}_{\mathrm{iK}}=2 \pi$.

The added mass matrix values with solving matrix equations for all six potential unit functions can be defined as follows:

$$
\mu_{\mathrm{iK}}=\rho \int_{\mathrm{S}_{\mathrm{B}}} \phi_{\mathrm{i}} \mathrm{n}_{\mathrm{K}} \mathrm{d} \mathrm{S}_{\mathrm{B}}
$$

If the above equation is written in the matrix form, which is the general form of added mass matrix. [12]:

$$
\begin{aligned}
& \text { Added Mass Matrix = }
\end{aligned}
$$

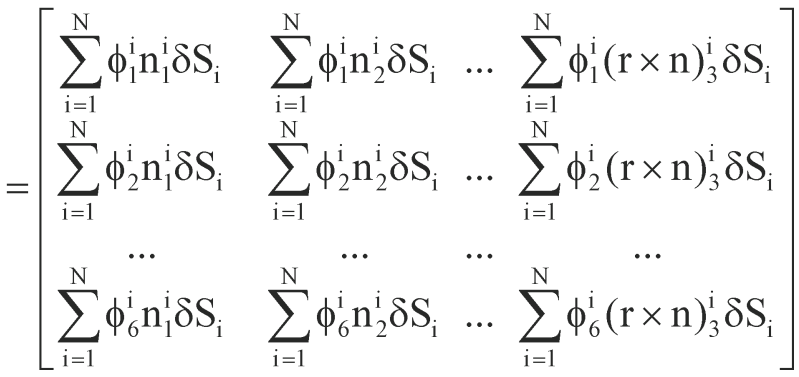

\section{RESULTS}

\section{Computing The Added Mass Coefficient of Sphere}

The added mass coefficient for a sphere of radius $0.25 \mathrm{~m}$ has been investigated in this part. However, according to the analytical relations in the mass matrix of sphere, only $a_{11}, a_{22}$ and $a_{33}$ have values and the others are zero. The three coefficients are equal with each other and should be equal to half of the sphere mass. In order to evaluate the Mesh independency, the added mass matrix were investigated for three cases of surface element (2400, 3360 and 4800 elements), using the boundary element numerical code and the obtained results and errors were checked.

The obtained values for the added mass of the sphere using numerical analysis of the boundary element are as follows:

Added Mass Matrix =

$=\left[\begin{array}{cccccc}0.032752 & 0 & 0 & 0 & 0 & 0 \\ 0 & 0.032753 & 0 & 0 & 0 & 0 \\ 0 & 0 & 0.032753 & 0 & 0 & 0 \\ 0 & 0 & 0 & 0 & 0 & 0 \\ 0 & 0 & 0 & 0 & 0 & 0 \\ 0 & 0 & 0 & 0 & 0 & 0\end{array}\right]$

For a sphere with radius of $0.25 \mathrm{~m}$, the analytical values of $\mathrm{a}_{11}, \mathrm{a}_{22}, \mathrm{a}_{33}$ are equal to 0.0327245

As it was mentioned, only $\mathrm{a}_{11}, \mathrm{a}_{22}, \mathrm{a}_{33}$ have non-zero value, which is equal to half of the sphere mass and the other elements of the added mass matrix are zero. So it can be seen that the obtained values of the numerical boundary element method have a high conformity with the analytical data and the error rate, according to the table (3) is the $10 \mathrm{E}-2$ order. [6]

Tab. 1. Geometric and flow data to calculate the added mass coefficient of sphere

\begin{tabular}{|l|}
\hline Radius $=0.25$ \\
Volume (Numerical) $=0.065449$ \\
Volume $($ Exact $)=0.0654498$ \\
Fluid $=$ Water \\
Density $=1000 \mathrm{~kg} / \mathrm{m}^{3}$
\end{tabular}

Tab. 2. Showing the produced geometry with surface elements to check the mesh independency condition

\begin{tabular}{|c|c|c|c|c|}
\hline $\begin{array}{l}\text { Number of } \\
\text { Element }\end{array}$ & $\begin{array}{c}\text { Numerical result for } \\
\mathbf{a}_{11} \text { (code result) }\end{array}$ & $\begin{array}{l}\text { Analytical } \\
\text { value of } a_{11}\end{array}$ & $\begin{array}{l}\text { Difference between } \\
\text { Numerical and Analytical result }\end{array}$ & $\begin{array}{c}\% \text { Error } \\
\left(\frac{a_{11(\text { Num })}-a_{11(\text { Anal })}}{a_{11(\text { Num })}}\right) \times 100\end{array}$ \\
\hline 2400 & 0.032814 & 0.032725 & $8.95 \mathrm{E}-05$ & 0.272749 \\
\hline 3360 & 0.032771 & 0.032725 & $4.65 \mathrm{E}-05$ & 0.141894 \\
\hline 4800 & 0.032752 & 0.032725 & $2.75 \mathrm{E}-05$ & 0.083964 \\
\hline
\end{tabular}

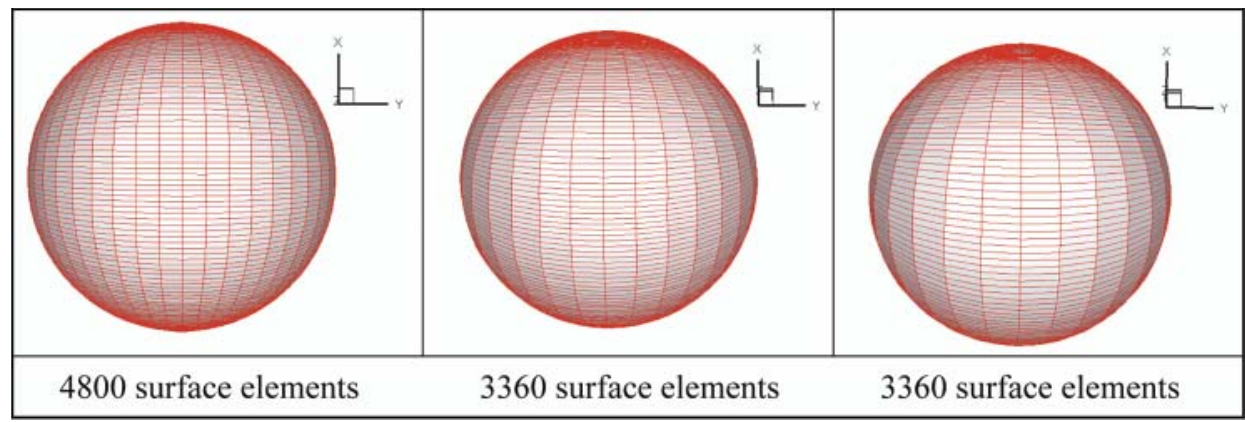

Tab. 3. The compared Results of the added mass numerical and analytical data of the sphere and the Error percentage Due to the elements numbers 
Tab. 4. Geometric and flow data of computing the added mass coefficient ellipsoid

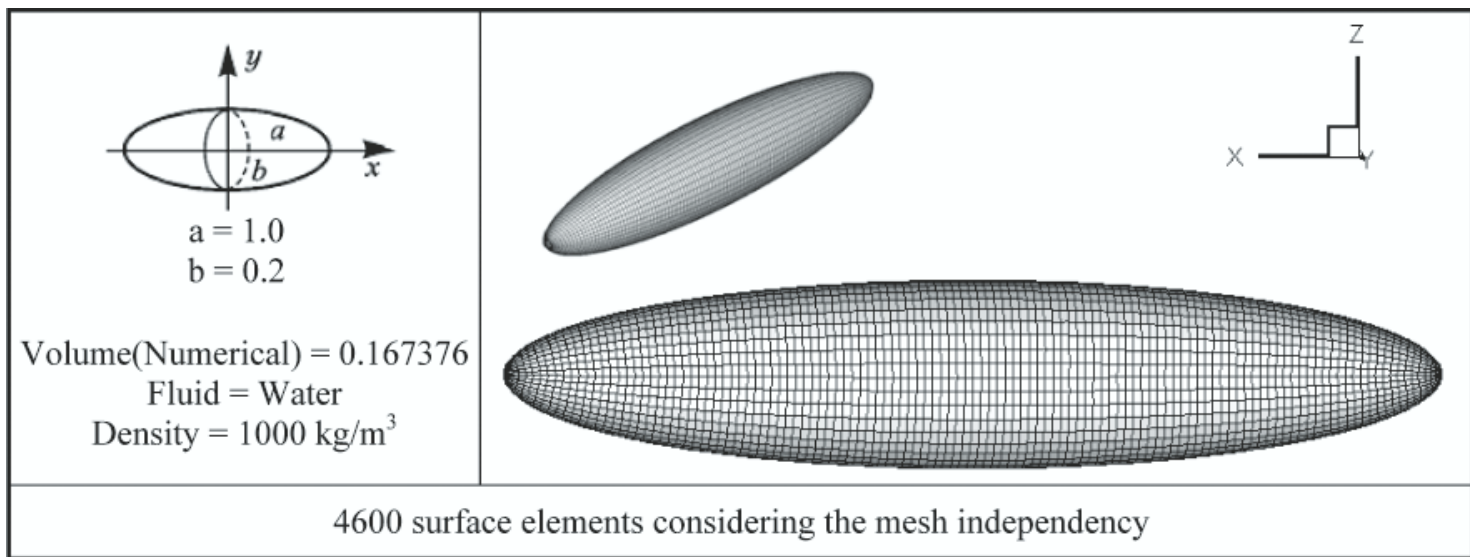

\section{Computing The Added Mass Of Ellipsoid}

Continue the contex, the coefficient of added mass for an ellipsoid has been investigated.

Since the added mass coefficients of ellipsoid as well as the sphere are available in references, so that to validate the computing results of the boundary element code, this analysis is given. The numerical analysis with the mesh independency condition was investigated and the results of the surface element with the number of 4600 had very little error in comparison with the existing data.

The added mass matrix obtained by numerical analysis for an ellipsoid with the above specification is as follows:

$$
\text { Added Mass Matrix = }
$$

$=\left[\begin{array}{cccccc}0.009025 & 0 & 0 & 0 & 0 & 0 \\ 0 & 0.143401 & 0 & 0 & 0 & 0 \\ 0 & 0 & 0.143401 & 0 & 0 & 0 \\ 0 & 0 & 0 & 0 & 0 & 0 \\ 0 & 0 & 0 & 0 & 0.030467 & 0 \\ 0 & 0 & 0 & 0 & 0 & 0.030467\end{array}\right]$

Considering that the numerical analysis results of the added mass were dimensional and the results in the references were dimensionless, then to validate the results, the data obtained from the numerical analysis were transformed to dimensionless one and compared with the available data in those references. To make the added mass matrix of ellipsoid dimensionless $\mathrm{a}_{11}$, $\mathrm{a}_{22}$ and $\mathrm{a}_{33}$ shall be divided on $(4 \pi / 3) \mathrm{ab}^{2}$ and also $\mathrm{a}_{44}, \mathrm{a}_{55}$ and $\mathrm{a}_{66}$ must be divided on $(4 \pi / 15) \mathrm{ab}^{2}\left(\mathrm{a}^{2}+\mathrm{b}^{2}\right)$.

Due to the symmetry of the ellipsoid, then $\mathrm{a}_{22}=\mathrm{a}_{33}, \mathrm{a}_{55}=\mathrm{a}_{66}$ and $\mathrm{a}_{44}=0$.

According to data obtained from the Diagram and making them dimensional, then:

$$
\begin{gathered}
a_{11}=K_{11} \times \frac{4 \pi}{3} a b^{2} \\
a_{22}=a_{33}=K_{22} \times \frac{4 \pi}{3} a b^{2} \\
a_{55}=a_{66}=K_{55} \times \frac{4 \pi}{15} a b^{2}\left(a^{2}+b^{2}\right)
\end{gathered}
$$

It is found from comparing the boundary element numerical analysis results with the data available in references for added mass coefficients of ellipsoid that there is a good conformity between the numerical data and the ones in the references $[6,12]$.

Tab. 5. The added mass coefficient Diagram of an ellipsoid [6]

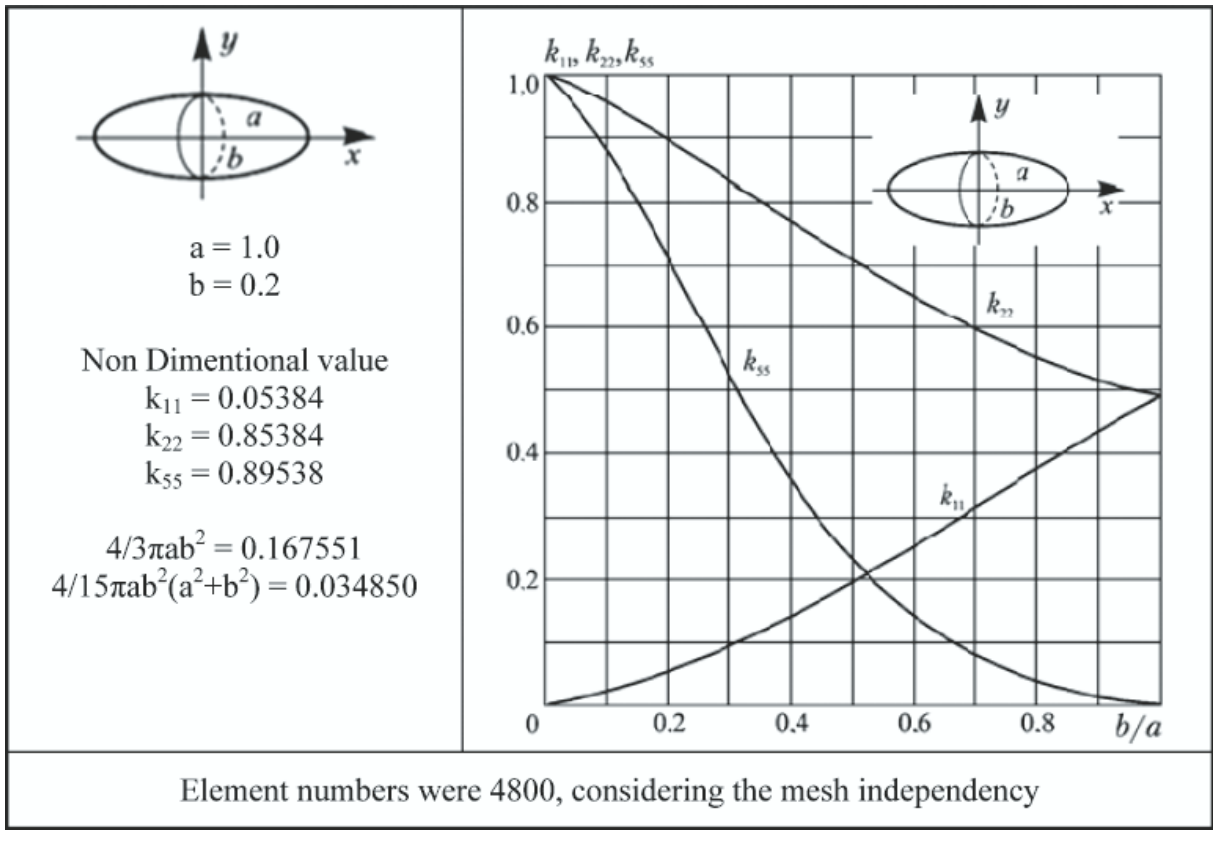


Tab. 6. the compared Results of the added mass numerical and analytical data of the ellipsoid and the Error percentage Due to the elements numbers

\begin{tabular}{|c|c|c|c|c|}
\hline $\begin{array}{c}\text { Matrix } \\
\text { array }\end{array}$ & $\begin{array}{c}\text { Numerical result } \\
\text { (code result) }\end{array}$ & $\begin{array}{c}\text { Analytical } \\
\text { result }\end{array}$ & $\begin{array}{c}\text { Difference between } \\
\text { Numerical and Analytical result }\end{array}$ & $\begin{array}{c}\mathbf{\%} \text { Error } \\
\left(\frac{\left.\mathbf{a}_{\mathbf{1 1}(\text { Num) }}-\mathbf{a}_{\mathbf{1 1}(\mathbf{A n a l})}\right) \times \mathbf{1 0 0}}{\mathbf{a}_{\mathbf{1 1}(\text { Num) }}}\right.\end{array}$ \\
\hline $\mathbf{A}_{\mathbf{1 1}}$ & 0.009025 & 0.00902094 & $4.06 \mathrm{E}-06$ & 0.044986 \\
\hline $\mathbf{A}_{\mathbf{2 2}}$ & 0.143401 & 0.14306174 & 0.000339 & 0.236581 \\
\hline $\mathbf{A}_{\mathbf{5 5}}$ & 0.030467 & 0.03120399 & -0.00074 & -2.41898 \\
\hline
\end{tabular}

\section{Computing The Added Mass Matrix For A Sample Propeller}

According to the data and results obtained for the added mass matrix of the sphere and ellipsoid it can be found that there is a very good accuracy between the data obtained from the analytical and the numerical solution, suggestive of the validity of the boundary element method code in calculating the added mass matrix. The following text is about computing the added mass matrix for a sample propeller which its experimental and geometrical data is on hand and the results have been validated with the experimental data.The added mass matrix, considering what was mentioned in the text do not depends on the body velocity and is only a function of the body geometry.

First of all, the existing Geometric data of the propeller was considered based on the standards and the propeller geometry modeled with a very high accuracy. Then the propeller surface and the hub were analyzed according to the flow conditions. The propeller analysis was performed in several different modes of surface element numbers so that the most appropriate numbers of the elements is achieved in a way that the obtained answers do not depend on the element numbers [14].

Tab. 7. Geometric and flow data related to the propeller and the produced geometry for boundary element numerical analysis [13]

\begin{tabular}{|l|c|}
\hline \multicolumn{1}{|c|}{ Property } & Value \\
\hline Type & FPP \\
\hline No. of Blades, $\mathrm{Z}$ & 5 \\
\hline Section Type & NACA66 \\
\hline Skew & $21.150 \mathrm{deg}$ \\
\hline Hub Ratio, H/D & 0.170 \\
\hline Pitch Ratio, P/D & 0.671 \\
\hline Expanded Area Ratio & 0.595 \\
\hline Advance Ratio, J & 0.4489 \\
\hline Diameter, D (m) & 0.25 \\
\hline Material & ALBC3 \\
\hline Young's Modulus, E (GPa) & 73 \\
\hline Shear Modulus, G (GPa) & 28 \\
\hline Material Density, $\rho \mathrm{mp}(\mathrm{kg} / \mathrm{m} 3)$ & 1,800 \\
\hline Water Density, $\rho w(\mathrm{~kg} / \mathrm{m} 3)$ & 487.4 \\
\hline Revolution Speed, $\mathrm{n}(\mathrm{rpm})$ & \\
\hline
\end{tabular}

Dimensional Added Mass Matrix Obtained For The Propeller

Added Mass Matrix $=\left[\begin{array}{cccccc}2.176192 & -0.240440 & -0.686280 & -0.670630 & 0.015638 & -0.196440 \\ -0.240440 & 0.042111 & 0.080637 & 0.006256 & 0.000156 & 0.018995 \\ -0.686280 & 0.080637 & 0.251128 & 0.019366 & -0.005240 & 0.059255 \\ -0.058640 & 0.006256 & 0.019366 & 0.001617 & -0.000450 & 0.005282 \\ 0.015638 & 0.000156 & -0.005240 & -0.000450 & 0.000505 & -0.001630 \\ -0.196440 & 0.018995 & 0.059255 & 0.005282 & -0.001630 & 0.018315\end{array}\right]$


Tab. 8. Compared results of the added mass numerical and analytical data of the propeller and the Error percentage Due to the element numbers

\begin{tabular}{|c|c|c|c|c|}
\hline $\begin{array}{l}\text { Number of } \\
\text { Element }\end{array}$ & $\begin{array}{l}\text { Numerical result } \\
\text { Matrix Array } \mathbf{A}_{13} \\
\text { (Addedprop code) }\end{array}$ & $\begin{array}{c}\text { Exprimental } \\
\text { Result }\end{array}$ & $\begin{array}{c}\text { Diffrence between } \\
\text { Numerical and Exprimental } \\
\text { result }\end{array}$ & $\left(\frac{a_{11(\text { Num })}-a_{11(A n a l)}}{a_{11(\text { Num })}}\right) \times 100$ \\
\hline 1800 & 0.6242060 & 0.6860000 & -0.0617940 & -9.0078717 \\
\hline 2520 & 0.6382161 & 0.6860000 & -0.0477839 & -6.9655831 \\
\hline 3240 & 0.6451033 & 0.6860000 & -0.0408967 & -5.9616181 \\
\hline 3960 & 0.6510313 & 0.6860000 & -0.0349687 & -5.0974781 \\
\hline 4320 & 0.6631462 & 0.6860000 & -0.0228538 & -3.3314577 \\
\hline 5040 & 0.6691640 & 0.6860000 & -0.0168360 & -2.4542274 \\
\hline 5760 & 0.6706359 & 0.6860000 & -0.0153641 & -2.2396647 \\
\hline
\end{tabular}

\section{Investigating The Mesh Independency}

To investigate that the obtained data for the propeller is independent of the produced surface elements of the propeller and the hub surface, the added mass matrix was obtained in seven different numbers of surface elements and the results were checked, as it is identified from the diagram (figure 2) the results do not have any significant change after 3960 elements.

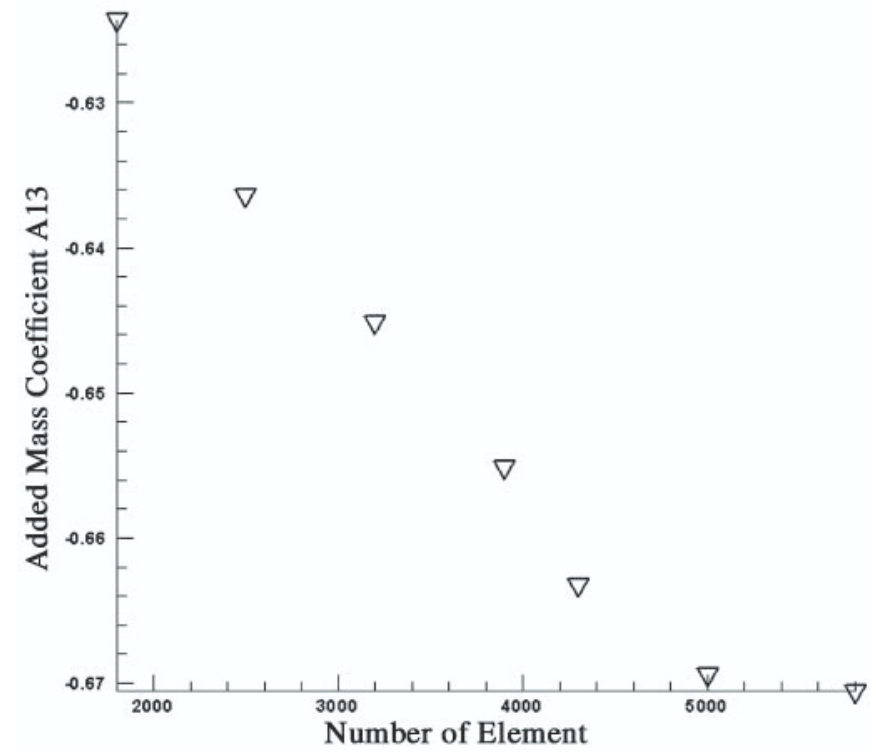

Fig. 2. Changes in the mass added coefficient based on the element numbers at the propeller surface

\section{Comparing The Added Mass Matrix Of The Propeller With The Obtained Data From Other Numerical And Experimental Methods}

Tab. 9. Comparing the data, obtained from the numerical solution, experimental and formulated data

\begin{tabular}{|c|c|c|}
\hline Estimation Method & $\begin{array}{c}\text { Added Mass } \\
\mathbf{( k g )}\end{array}$ & $\begin{array}{c}\text { Diff } \\
{[\mathbf{\%}]}\end{array}$ \\
\hline Experiment & 0.6860000 & 0.0 \\
\hline AddedProp (Present method) & 0.6706359 & $-2.24 \%$ \\
\hline Prodas & 0.6690000 & $-2.5 \%$ \\
\hline Schwanecke & 0.8240000 & $+20.1 \%$ \\
\hline Shuster & 0.9660000 & $+40.8 \%$ \\
\hline
\end{tabular}




\section{Computing The Added Mass Matrix Of The Hydrofoil (NACA0012 Section)}

Tab. 10. Geometrical data and the element numbers of calculating the added mass coefficient of hydrofoil

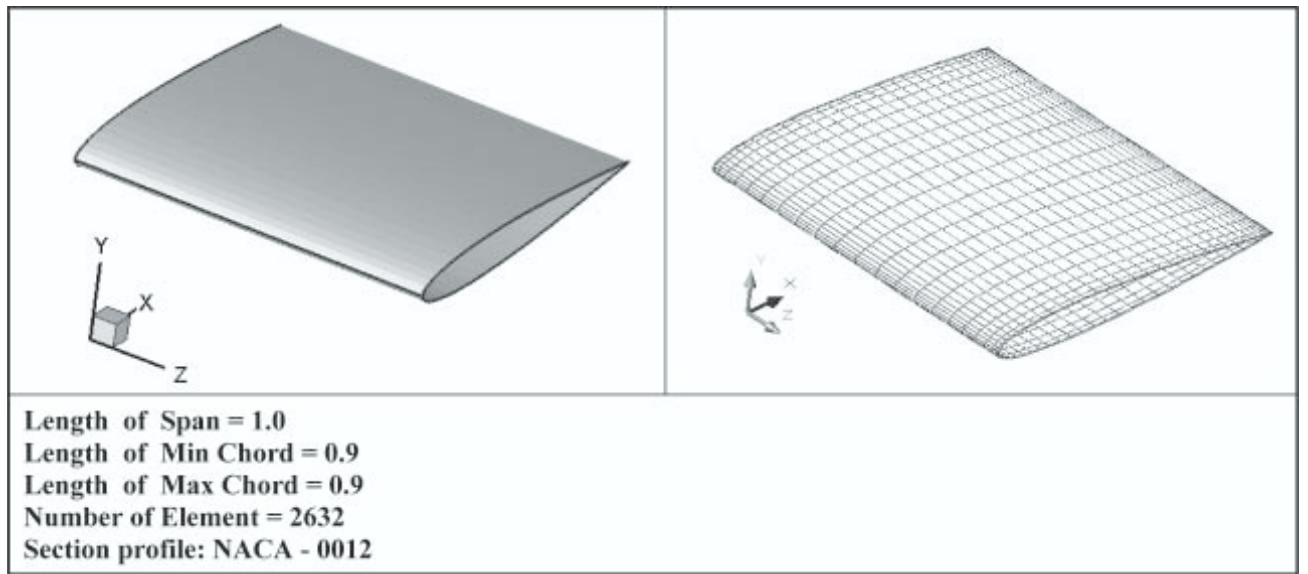

\section{Gradual Increase In The Span Length To Validate The Results}

To calculate the added mass matrix of the hydrofoil with NACA0012 section, first of all the mesh independency was investigated in which the obtained data from 2632 elements had very good accuracy, then to validate the obtained data, the span length was increased gradually from 0.2 to 1 and thus the added mass matrix was computed. Afterward the added mass matrix element changes were plotted based on the span length increase. All of the coefficients are increased with linear and non-linear curves of the 2 and 3 order, which is completely ascending. In fact, by increasing the Span length, all coefficients were reasonably increased as expected. Of course, investigating each of the diagrams whether they should be linear or nonlinear, of the 2 orders or 3 orders are denied due to that they are Proportional to Dynamic and Simulation topics.

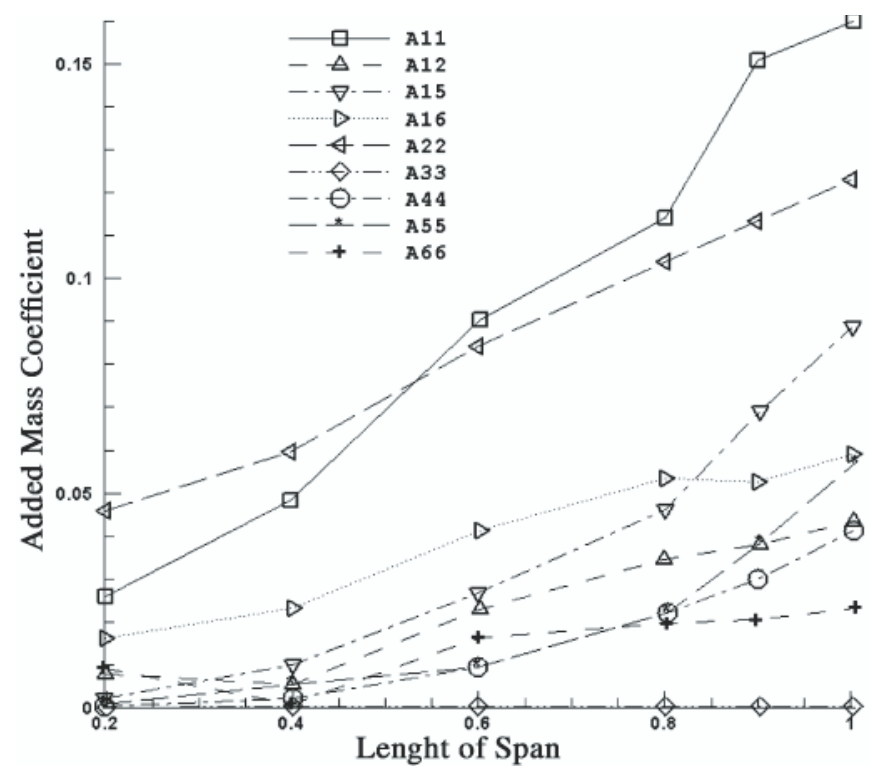

Fig. 3. The added mass coefficient changes of hydrofoil based on the gradually increase along the Span length

\section{The Added Mass Matrix Of Hydrofoil}

The added mass matrix of hydrofoil with NACA0012 section for the Span length of $1 \mathrm{~m}$ and Chord length of 9/0 m is as follows:

Added Mass Matrix $=\left[\begin{array}{cccccc}159.3152 & 31.63824 & 0 & -13.5806 & 89.37951 & 58.75352 \\ 31.63824 & 122.4136 & 0 & -63.4481 & 11.35911 & 44.82613 \\ 0 & 0 & 0 & 0 & 0 & 0 \\ -13.5806 & -63.4481 & 0 & 40.92427 & -7.51047 & -23.5442 \\ 89.37951 & 11.35911 & 0 & -7.51047 & 56.3155 & 21.46682 \\ 58.75352 & 44.82613 & 0 & -23.5442 & 21.46682 & 22.75078\end{array}\right]$




\section{CONCLUSION}

- The added mass coefficient is one of the most important dynamic coefficients in solving dynamic equations and simulating the vessels. Calculating the Add mass coefficient especially the bodies whose shapes are not simple is difficult. Added mass coefficient is extractable using empirical formulas, the simple analytical relations and numerical methods such as Strip theory and lab tests.

- But each of these methods can be used in a limited range. For example, the analysis relations can be used only for simple geometries and lab tests to extract the added mass are costly and obtaining all matrix coefficients is very difficult. Moreover, the numerical methods cannot model the full three-dimensional body and have a lot of errors that ultimately causing a large deviation in calculating the coefficients. Meanwhile since the boundary element method solves the problem on surface boundary elements of the body, it needs less elements and time to solve and can obtain the added mass coefficients owing to modeling the full three-dimensional body and also extracting the potential values with high accuracy.

- As mentioned in the article to investigate the mesh independency, the added mass matrix of an ellipsoid and a sphere of specific geometry were extracted, using the boundary element method and the results were validated with the analytical data.

- Afterward the added mass matrix for a certain propeller was computed which its results had a very good conformity with the experimental ones. However due to lack of access to distribution Cord of the propeller, the standard distribution of Series B for a propeller with specific section (NACAa66) was used, considering that the volume of the produced propeller geometry was checked with the volume of the examined propeller in a way that the geometry would have a high conformity with the existing data so that if the exact geometry is on hand, the error rate with be lower than 10E-2 order.

- To put it in a nut shell, extracting a complete and dimensional add mass matrix with any desired geometry and the accuracy of obtained data is the difference between this method and boundary element code with other numerical methods.

\section{BIBLIOGRAPHY}

1. Newman, John Nicholas: Marine hydrodynamics. Cambridge, Massachusetts, MIT Press. ISBN: 0-262-14026-8 §4.13, p. 139, 1977
2. Birkhoff, G.: Hydrodynamics. Princeton Univ. Press, Princeton, 1960

3. Lamb, G.: Hydrodynamics. Cambridge University Press, Cambridge, 1932

4. Kochin, N.E., Kibel, I.A., Rose, N.V.: Theoretical Hydromechanics, Parts I and II. State Publisher of Physical and Mathematical Literature, Moscow, in Russian, 1963

5. Taylor, J.L.: Some hydrodynamical inertia coefficients. Phil. Mag. Ser. 7 9(55), 161-183, 1930

6. Alexandr I. Korotkin: Added mass of ship structure. Fluid mechanics and its applications, Volume 88, 2009

7. John T. Katsikadelis: Boundary Elements, Theory and Applications, Elsevier Science Ltd, 2002

8. Kinnas S.A. and Hsin C.Y.: Boundary Element Method for the Analysis of the Unsteady Flow around Extreme Propeller Geometry. IAA, Journal, 30 (3), 1992

9. Hess J.T, Smith A.M.: Lecture 3 AA200b January 13-18, 2005

10.Hess J.T, Smith A.M.: Calculation of nonlifting potential flow about arbitrary three-dimensional bodies. Journal of Ship Research, 8(2), 1964

11.Morino L. and Kuo C.C.: Subsonic Potential Aerodynamics for Complex Configuration: A general Theory. AIAA Journal, 12 (2), 191-197, 1974

12.Olivier Saout.: Computation of Hydrodynamic Coefficients and Determination of Dynamic Stability Characteristics of an Underwater Vehicle Including Free Surface Effects. Ms Thesis,Florida Atlantic University, May 2003

13. Young-Joong Kim, Hyun Yup Lee and Chang-Sup Lee: The Added Mass and Damping for the Axial Rigid Body Motion of a Marine Propeller Rotating in a Uniform Flow. Journal of the Society of Naval Architects of Korea, Vol. 45, No. 3, pp. 309-314, June 2008

14.Donald M. MacPherson and Vincent R. Puleo and Matthew B. Packard, Estimation of Entrained Water Added Mass Properties for Vibration Analysis, SNAME New England Section, June 2007.

\section{CONTACT WITH THE AUTHORS}

Hassan Ghassemi, Assoc. Prof. Faculty of Marine Technology Amirkabir University of Technology Hafez Ave., Tehran, IRAN

e-mail: gasemi@cic.aut.ac.ir

Ehsan Yari, M. Sc.

Faculty of Marine Engineering, Malekashtar University of Technology, Shahinshar, Isfahan, IRAN 